\title{
Clinical Study \\ Height Constitutes an Important Predictor of Mortality in End-Stage Renal Disease
}

\author{
Tsuneo Takenaka, Takahiko Sato, Hitoshi Hoshi, Nobutaka Kato, Keita Sueyoshi, \\ Masahiro Tsuda, Yusuke Watanabe, Hiroshi Takane, Yoichi Ohno, and Hiromichi Suzuki
}

Department of Medicine, Saitama Medical College, 38 Moro-hongo, Moroyama, Iruma, Saitama 395-0495, Japan

Correspondence should be addressed to Tsuneo Takenaka, takenaka@saitama-med.ac.jp

Received 16 July 2010; Revised 20 September 2010; Accepted 2 October 2010

Academic Editor: Ken Ichi Aihara

Copyright (C) 2011 Tsuneo Takenaka et al. This is an open access article distributed under the Creative Commons Attribution License, which permits unrestricted use, distribution, and reproduction in any medium, provided the original work is properly cited.

\begin{abstract}
Aim. Height is an important determinant of augmentation index (AI) that anticipates cardiovascular prognosis. There is a scanty of the data whether short height predicts survival in patients with end-stage renal diseases, a high risk population. Methods. Fifty two hypertensive patients with type 2 diabetic nephropathy receiving hemodialysis and 52 patients with nondiabetic nephropathy were enrolled. In addition to AI estimated with radial artery tonometry, classical cardiovascular risk factors were considered. Patients were followed for 2 years to assess cardiovascular prognosis. Results. Cox hazards regression revealed that both smoking and shortness in height independently contributed to total mortality and indicated that smoking as well as the presence of left ventricular hypertrophy predicted cardiovascular mortality. Our findings implicated that high AI, the presence of diabetes, and low high-density lipoprotein cholesterol were significant contributors to cardiovascular events. Conclusions. Our findings provide new evidence that shortness in height independently contributes to total mortality in hemodialysis patients.
\end{abstract}

\section{Introduction}

Recent technical advance enables noninvasive screening of arterial stiffness, and an increase in arterial stiffness is a character of atherosclerosis [1-9]. Pulse wave velocity (PWV) and augmentation index (AI) are good examples. Both PWV and AI predict cardiovascular survival in patients with hypertension or renal insufficiency [4-9]. Recently, ASCOTCAFE study has clearly demonstrated that an elevated augmentation index (AI) is an important cardiovascular risk in hypertensive patients [9]. Although PWV could predict cardiovascular mortality in diabetes [5], there is scanty of data on AI. Furthermore, available data in diabetes failed to provide a consistent pattern. On the one hand, Lacy et al. demonstrated that diabetes showed an elevated PWV but a normal AI [10]. On the other hand, Westerbacka et al. reported that AI was associated with carotid intimamedia thickness in diabetes [11]. AI is a dynamic index of arterial stiffness, reflecting not only elastic but also muscular artery stiffness $[12,13]$. The stiffness of muscular artery is modulated by vasoactive agents including nitric oxide, angiotensin II, and autonomic nerve activity. Collectively, variations in these factors may elicit changes in AI. Indeed, we have demonstrated that AI in diabetes shows abnormal regulations following acute postural changes [14].

There is a debate on the relationship between height and cardiovascular disease. A recent meta-analysis demonstrated that short statue is associated with coronary heart disease [15]. In contrast, studies from South Korea indicated that height inversely related to mortality in both men and women without an association with coronary heart disease $[16,17]$. Lee et al. reported an opposing relation of height with death from cardiovascular diseases and cancers among people in Asia-Pacific area [18]. Various mechanisms underlying this relation are proposed such as poor nutrition during childhood, increased LDL cholesterol, genetic liability, high pulse pressure, and systolic blood pressure in adults with short height [15-21]. Indeed, systolic blood pressure is suited to assess cardiovascular risk in Japanese [22]. Of interest, height is inversely related to $\mathrm{AI}$, and high $\mathrm{AI}$ is 


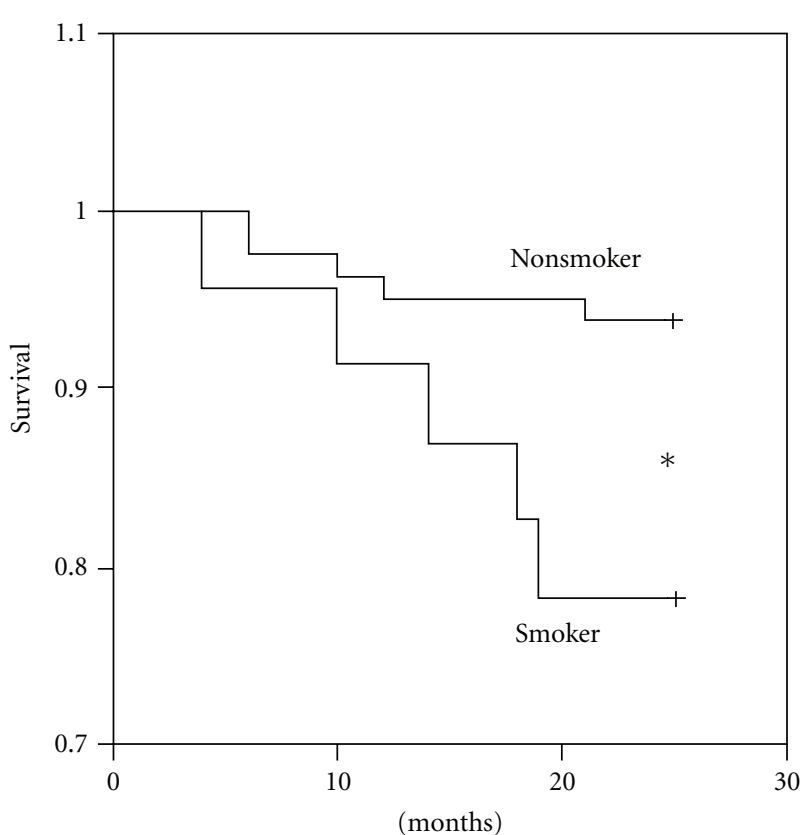

Figure 1: Kaplan-Meier analysis on whole hemodialysis patients showed that nonsmokers survived better than smokers. Log-rank test denoted that there was a significant difference in mortality between smoker and nonsmoker.

a cardiovascular risk [9]. Cardiovascular diseases are major causes of death in hemodialysis patients [23]. The present study was performed to assess whether short height predicts mortality in renal patients.

Diabetes as well as hypertension is well-known cardiovascular risk factors. Recent data indicated that cardiovascular events occurred three times more frequently in patients with chronic kidney diseases [24]. Diabetes is now a primary disease, which requires renal replacement therapy $[5,25]$. Moreover, although dialysis therapy saves their lives, it may also burden additional cardiovascular risks to them. In the present study, the relationship between cardiovascular risk factors including AI and cardiovascular prognosis was examined in hypertensive patients with diabetic and nondiabetic nephropathy receiving regular dialysis therapy. Our data provide the evidence that left ventricular hypertrophy $(\mathrm{LVH})$ predicts cardiovascular events in diabetic patients undergoing hemodialysis. In addition, the present results indicate that nondiabetic patients with higher AI suffered from more cardiovascular events. Our findings implicate that smoking and LVH predict cardiovascular death, and further suggest that in addition to smoking, short statue is a risk for mortality in hemodialysis patients.

\section{Methods}

Patients who visited our offices to perform chronic hemodialysis were listed for the study when they accept the informed consent. The following patients were not enrolled: patients with myocardial infarction or stroke within

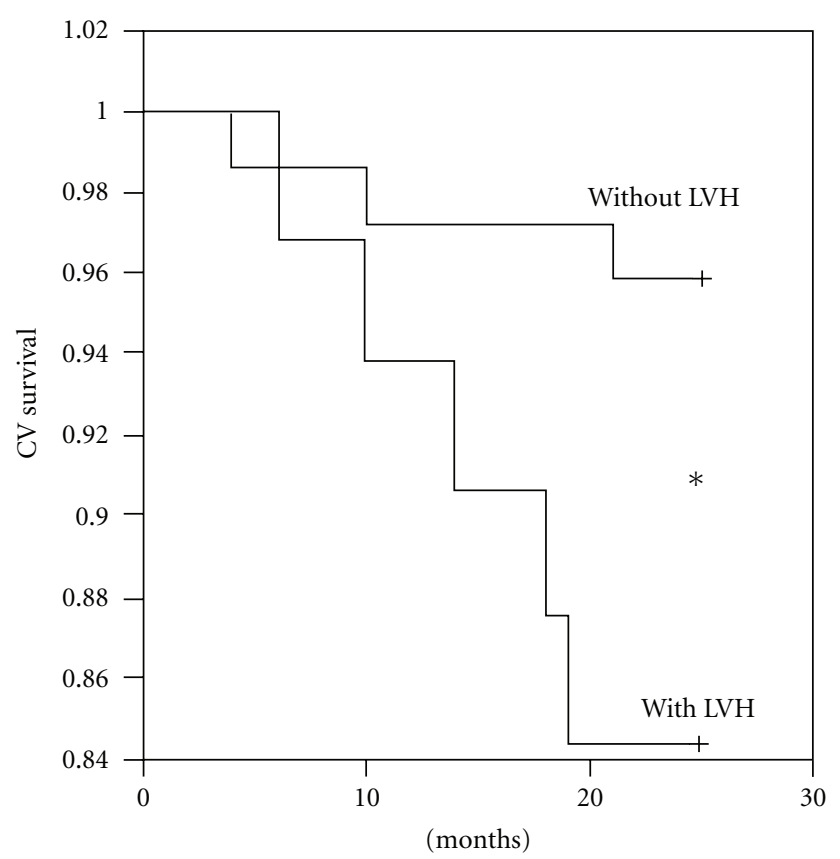

Figure 2: Kaplan-Meier analysis on whole hemodialysis patients showed that those without left ventricular hypertrophy (LVH) suffered cardiovascular death less than those with LVH. Log-rank test denoted that there was a significant difference in cardiovascular mortality between patients with LVH and those without.

6 months, patients with unstable angina pectoris, patients with persistent arrhythmia, patients with heart failure or left ventricular ejection fraction of $40 \%$ or less, patients with peripheral artery disease (stage 3-4), and patients with overt secondary hyperparathyroidism [26]. Patient entry was started in 2003, and 52 type II diabetic patients with nephropathy entered the study. Age- and sex-matched 52 nondiabetic hemodialysis patients were enrolled as controls to perform case-control study. All 104 patients received hemodialysis therapy for 4 hours, 3 days a week. Dry weight was carefully determined for an individual patient to achieve edema-free state in references to blood pressure and cardiac thorax ratio. In selected patients, enddiastolic left ventricular diameter, inferior vena cava diameter, and plasma level of atrial natriuretic peptide were also assessed.

Radial AI was assessed using automated tonometry (HEM9010-AI, Omron Healthcare). This device was designed to automatically record pulse waves using series of tonometric sensors faced on radial artery. AI was determined by dividing reflection pressure by ejection pressure with variations of $4.4 \%$ [27]. This device also measures blood pressure using an oscillometric method. Two measurements were performed with the patient in the sitting position for 5 and 10 minutes, respectively, and the average of the 2 values was taken for the purpose efficacy analysis. This device was able to estimate central blood pressure expressed as SBP2 [28]. Electrocardiogram and blood samples were taken from all patients at the time of entry. The following criteria were used to diagnose LVH; Sokolow-Lyon voltage 


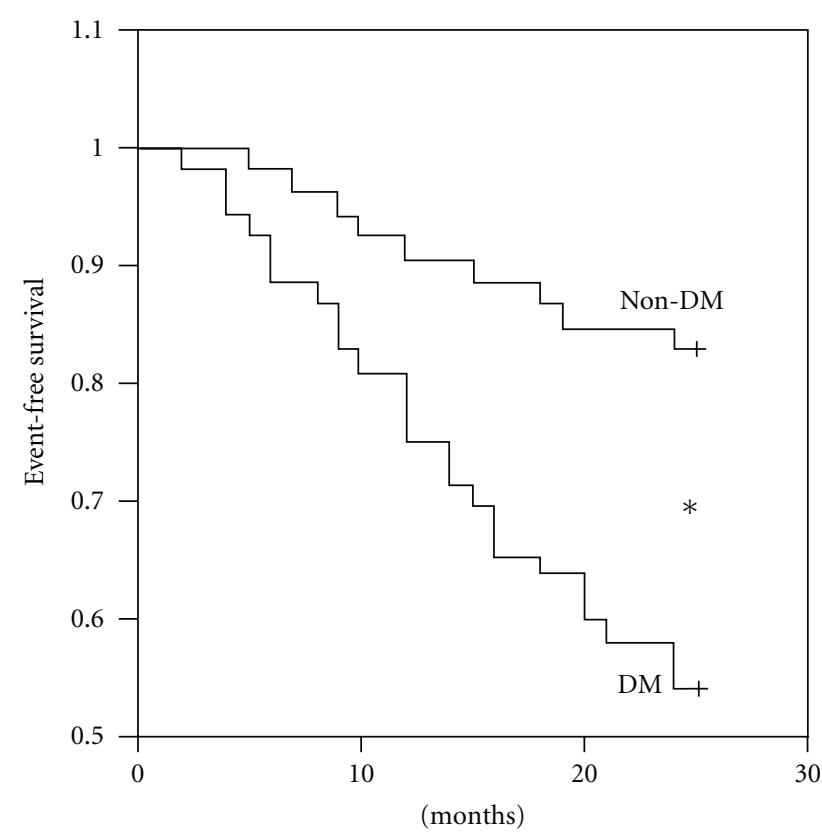

FIgURE 3: Kaplan-Meier analysis on whole hemodialysis patients showed that those without diabetes experienced cardiovascular events less frequently than those with diabetes. Log-rank test depicted that there was a significant difference in cardiovascular events between patients with diabetes and those without.

amplitude of (SV1 + RV5 or RV6) > $3.5 \mathrm{mV}$, a Cornell voltage of $(\mathrm{SV} 3+\mathrm{RaVL})>2.8 \mathrm{mV}$, or a Cornell product of $[(\mathrm{SV} 3+\mathrm{RaVL}) \times \mathrm{QRS}$ duration $]>244 \mathrm{mV}$. Left ventricular strain was defined as a down-sloping ST-segment depression $>0.1 \mathrm{mV}$ with T-wave flattening or inversion in leads V4 to V5 [29]. Blood sample was drawn after overnight fast for accuracy.

The patients were followed for 2 years or until cardiovascular events occurred. Cardiovascular events included coronary artery disease, stroke, and peripheral artery disease. Heart failure was excluded because pulmonary edema only due to fluid retention is common in this patient population [30]. Thus, the following patients were considered to possess cardiovascular events: patients with acute myocardial infarction, those with angina pectoris being treated by either bypass surgery or balloon angioplasty, patients with brain infarction or bleeding, and patients with peripheral artery disease who received limb amputation, balloon angioplasty, or bypass surgery.

Cox hazard analysis with Wald modification was performed to assess cardiovascular risk. In addition to AI, the presence or absence of LVH and diabetes, classical Flamingham risk factors including age, sex, height, weight, mean blood pressure (MBP), pulse rate (PR), total cholesterol (TC), high-density lipoprotein cholesterol (HDL-C), triglyceride (TG), and current smoking were used as independent variables. Nonclassical risk factors such as serum albumin, hemoglobin $(\mathrm{Hb})$, hemodialysis duration, and KT/V were also considered as independent variables. AI showed significant relationship with SBP and SBP2 $(P<.01)$. Since we

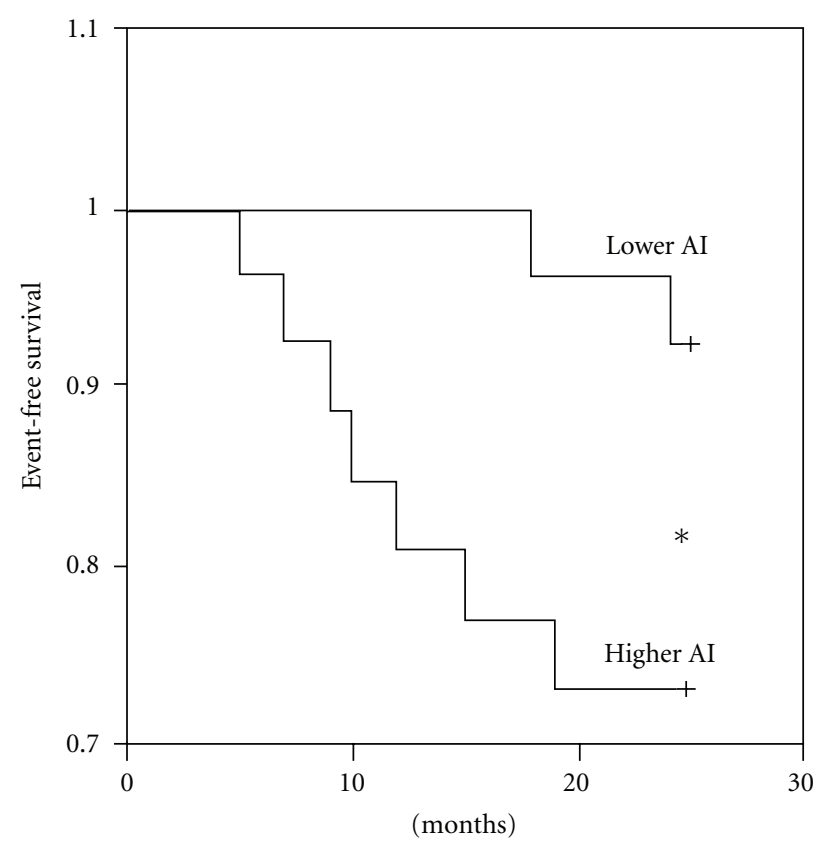

FIGURE 4: Kaplan-Meier analysis on nondiabetic hemodialysis patients showed that those with higher AI failed to live cardiovascular event-free lives similar to those with the lower AI. Log-rank test denoted that there was a significant difference in cardiovascular events between two subgroups.

used AI, an index of arterial stiffness, as an independent variable, MBP was selected as an independent variable of blood pressure component to decrease multi-co-linearity [31]. Kaplan-Meier analysis was performed to assess a contributor to cardiovascular events. Cox hazard model was also applied to consider cardiovascular risk in diabetic and nondiabetic groups, respectively. Age, sex, height, weight, MBP, PR, TC, HDL-C, TG, AI, the presence or absence of $\mathrm{LVH}$ and smoking were used as independent variables in subgroup analysis. The median of AI and HDL-C was used to divide patients into two groups. Stepwise regression analysis was used to assess which patient backgrounds determined AI. Receiver operation characteristic (ROC) analysis was used to assess cut-off value of AI to predict cardiovascular events. Fisher's exact test and Student t-test were used to compare patient background between groups. $P<.05$ was considered significant.

\section{Results}

Table 1 summarized patient background for two groups. In each group, there were 11 females and age was averaged $59 \mathrm{y} / \mathrm{o}$ at the time of study entry. Height, weight, MBP, $\mathrm{PR}, \mathrm{AI}, \mathrm{TC}, \mathrm{HDL}-\mathrm{C}$, and the prevalence of smokers were similar between nondiabetic and diabetic groups. Twenty diabetic patients were on insulin therapy, and 10 took voglibose. The other 22 diabetes were diet therapy alone. Averaged HbA1c was $6.1 \pm 0.3 \%$ in diabetes. LVH was more frequently observed in diabetes than nondiabetics, and TG was higher in diabetes. Although serum albumin 


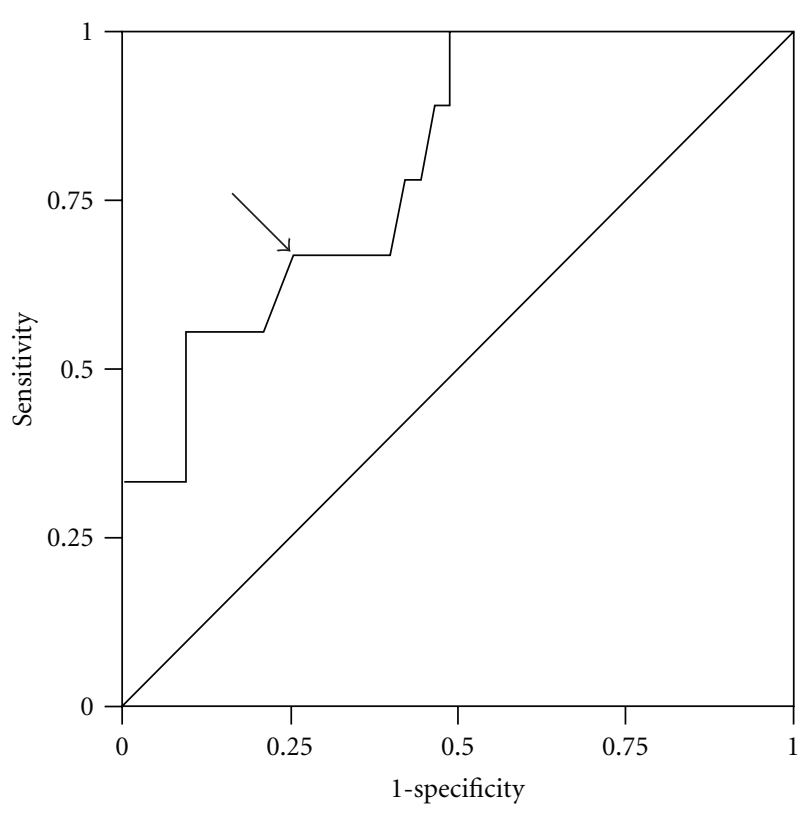

Figure 5: ROC curve for nondiabetic patients showed that AI of 87 was able to be used as a cutoff value to (arrow) predict cardiovascular events.

$(3.8 \pm 0.3$ versus $3.8 \pm 0.2 \mathrm{~g} / \mathrm{dl}), \mathrm{Hb}(10.0 \pm 0.5$ versus $9.9 \pm 0.6 \mathrm{~g} / \mathrm{dl})$, and dialysis efficiency $(\mathrm{KT} / \mathrm{V} ; 1.1 \pm 0.1$ versus $1.1 \pm 0.1$ ) were similar between 2 groups, the duration of hemodialysis in diabetes was shorter than that of nondiabetic patients. Most patients took antihypertensives. Either an angiotensin converting enzyme inhibitor or an angiotensin receptor blocker was prescribed for $87 \%$ of diabetic patients and $77 \%$ of nondiabetic patients. Statins were prescribed for $58 \%$ of diabetic patients and $54 \%$ of nondiabetic patients. No patients took fibrates, because Ministry of Health, Labor and Welfare Japan prohibited their application for patients with renal insufficiency. Erythropoietin was administered to treat renal anemia and titrated to maintain hemoglobin around $10 \mathrm{~g} / \mathrm{dl}$. The doses of erythropoietin were similar between diabetic $(3800 \pm 435 \mathrm{IU} /$ week $)$ and nondiabetic patients $(3950 \pm 383 \mathrm{IU} /$ week).

Three patients died during 2 years in the nondiabetic group. The causes of death were stroke (2 cases) and gastric cancer. Eight diabetic patients died during observation periods. Three diabetic patients were dead from myocardial infarction, two from stroke, and one from peripheral artery diseases. The other two patients died from renal cell carcinoma and pneumonia. Thus, only $5 \%$ of patients were dead for a year in the present study. As with previous reports, our results support a significant small death rate of hemodialysis patients in Japan, compared to US or Europe [32].

Cox hazard analysis demonstrated that both smoking $(P<.05)$ and shortness in height $(P<.05)$ considerably contributed to total mortality (Table 2 ). Figure 1 depicted Kaplan-Meier analysis indicating a significant difference in survival curves between smokers and nonsmokers $(P<.05)$. Cox hazard model selected both smoking $(P<.05)$ and LVH $(P<.05)$ as significant contributors to cardiovascular
TABle 1: Demographic feature of participating patients.

\begin{tabular}{lcc}
\hline & Non-DM & DM \\
\hline Age (y/o) & $59 \pm 13$ & $59 \pm 12$ \\
Sex (M/F) & $41 / 11$ & $41 / 11$ \\
height (cm) & $164 \pm 9$ & $163 \pm 8$ \\
Weight (kg) & $60 \pm 10$ & $60 \pm 11$ \\
SBP (mmHg) & $143 \pm 19$ & $146 \pm 20$ \\
DBP (mmHg) & $69 \pm 13$ & $67 \pm 16$ \\
SBP2 (mmHg) & $127 \pm 16$ & $129 \pm 18$ \\
MBP (mmHg) & $94 \pm 12$ & $94 \pm 13$ \\
PR (bpm) & $78 \pm 18$ & $79 \pm 10$ \\
TC (mg/dl) & $157 \pm 42$ & $158 \pm 36$ \\
HDL-C (mg/dl) & $40 \pm 15$ & $36 \pm 11$ \\
TG (mg/dl) & $115 \pm 45$ & $134 \pm 52^{*}$ \\
Smoking (\%) & 19 & 25 \\
AI & $78 \pm 21$ & $79 \pm 20$ \\
LVH (\%) & 15 & $46^{*}$ \\
HD duration (yrs) & $12.4 \pm 1.0$ & $9.6 \pm 0.8^{*}$ \\
\hline
\end{tabular}

DM, HD, PR, TC, HDL-C, TG, AI, LVH, SBP, SBP2, DBP, and MBP depicted diabetes mellitus, hemodialysis, pulse rate, total cholesterol, high density lipoprotein cholesterol, triglyceride, augmentation index, left ventricular hypertrophy, systolic, central, diastolic and mean blood pressure, respectively. ${ }^{*}$ indicates significance between groups.

TABLe 2: Cox hazard stepwise regression analysis for all mortality (whole patients). $\chi^{2}=8.54, d f=2, P=.014$.

\begin{tabular}{lcccc}
\hline Variable & $\beta$ & SE & Wald & Probability \\
\hline Smoking & 1.65 & 0.65 & 6.35 & 0.012 \\
Height & -0.082 & 0.038 & 4.80 & 0.028 \\
\hline
\end{tabular}

SE; standard error.

TABLE 3: Cox hazard stepwise regression analysis for cardiovascular mortality (whole patients). $\chi^{2}=10.22, d f=2, P=.006$.

\begin{tabular}{lcccc}
\hline Variable & $\beta$ & SE & Wald & Probability \\
\hline Smoking & 1.858 & 0.731 & 6.45 & 0.011 \\
LVH & 1.372 & 0.713 & 3.52 & 0.041 \\
\hline
\end{tabular}

SE and LVH indicate standard error and left ventricular hypertrophy.

mortality (Table 3). As shown in Figure 2, patients with LVH survived less than those without $(P<.05)$. As shown in Table 4, however, Cox hazard analysis showed that the presence of diabetes $(P<.05)$, high AI $(P<.05)$, and low HDL-C $(P<.05)$ contributed to cardiovascular events in this study. Figure 3 depicted that diabetic patients suffered more cardiovascular events than nondiabetic patients $(P<.05)$.

Subgroup analysis was also performed. In nondiabetic patients, Cox hazard model failed to find significant contributors to total and cardiovascular mortality. However, it selected high AI as a contributor to cardiovascular events (Table 5). Figure 4 exhibits Kaplan Meier analysis which showed that patients with low AI enjoy cardiovascular eventfree lives longer than those with high AI. As illustrated in Figure 5, ROC analysis has shown that the cutoff value of 
TABLE 4: Cox hazard stepwise regression analysis for cardiovascular events (whole patients). $\chi^{2}=23.3, d f=3, P=.0001$.

\begin{tabular}{lcccc}
\hline Variable & $\beta$ & SE & Wald & Probability \\
\hline AI & 0.026 & 0.009 & 8.499 & 0.004 \\
HDL-C & -0.045 & 0.018 & 6.208 & 0.013 \\
DM & 1.155 & 0.394 & 8.593 & 0.003 \\
\hline
\end{tabular}

SE, AI, HDL-C, and DM depicted standard error, augmentation index, high density lipoprotein cholesterol, and diabetes mellitus.

TABLE 5: Cox hazard stepwise regression analysis for cardiovascular events (nondiabetic patients). $\chi^{2}=11.42, d f=1, P=.001$.

\begin{tabular}{lcccc}
\hline Variable & $\beta$ & SE & Wald & Probability \\
\hline AI & 0.064 & 0.021 & 9.090 & 0.003 \\
\hline
\end{tabular}

SE and AI described standard error and augmentation index.

TABLE 6: Cox hazard stepwise regression analysis for cardiovascular events (diabetic patients). $\chi^{2}=10.07, d f=2, P=.007$.

\begin{tabular}{lcccc}
\hline Variable & $\beta$ & SE & Wald & Probability \\
\hline HDL-C & -0.047 & 0.021 & 4.819 & 0.028 \\
LVH & 0.987 & 0.425 & 5.405 & 0.020 \\
\hline
\end{tabular}

SE, HDL-C, and LVH denoted standard error, high density lipoprotein cholesterol, and left ventricular hypertrophy.

TABLE 7: Stepwise regression analysis for AI (nondiabetic patients). $R^{2}=0.428$, RMSE $16.52, F=18.33, P<.001$.

\begin{tabular}{lcccc}
\hline Variable & $\beta$ & SE & $t$ & $P$ \\
\hline Age & 0.644 & 0.198 & 3.249 & 0.002 \\
Height & -0.926 & 0.306 & -3.029 & 0.004 \\
\hline
\end{tabular}

$\mathrm{AI}$ and SE represented augmentation index and standard error.

TABle 8: Stepwise regression analysis for AI (diabetic patients). $R^{2}=0.303$, RMSE 16.58, $F=10.65, P<.001$.

\begin{tabular}{lcccc}
\hline Variable & $\beta$ & SE & $t$ & $P$ \\
\hline MBP & 0.404 & 0.178 & 2.268 & 0.028 \\
Height & -1.140 & 0.289 & -3.940 & 0.001 \\
\hline
\end{tabular}

AI, SE, and MBP indicated augmentation index, standard error, and mean blood pressure.

87 for AI predicts the occurrence of cardiovascular events in nondiabetic hemodialysis patients with sensitivity and specificity of $67 \%$ and $74 \%$, respectively. In diabetic patients, Cox hazard model failed to find significant contributors to total and cardiovascular mortality as the case with nondiabetic patients. However, Cox hazard analysis chose the presence of LVH $(P<.05)$ and low HDL-C $(P<.05)$ as the predictors of cardiovascular events (Table 6). Figure 6 demonstrated that hemodialysis patients with the lower HDL-C failed to live cardiovascular event-free lives similar to those with the higher HDL-C. Stepwise regression analysis on patient backgrounds in each group described that age and height significantly contributed to AI in nondiabetic group (Table 7) and showed that MBP and height determined $\mathrm{AI}$ in diabetic group (Table 8).

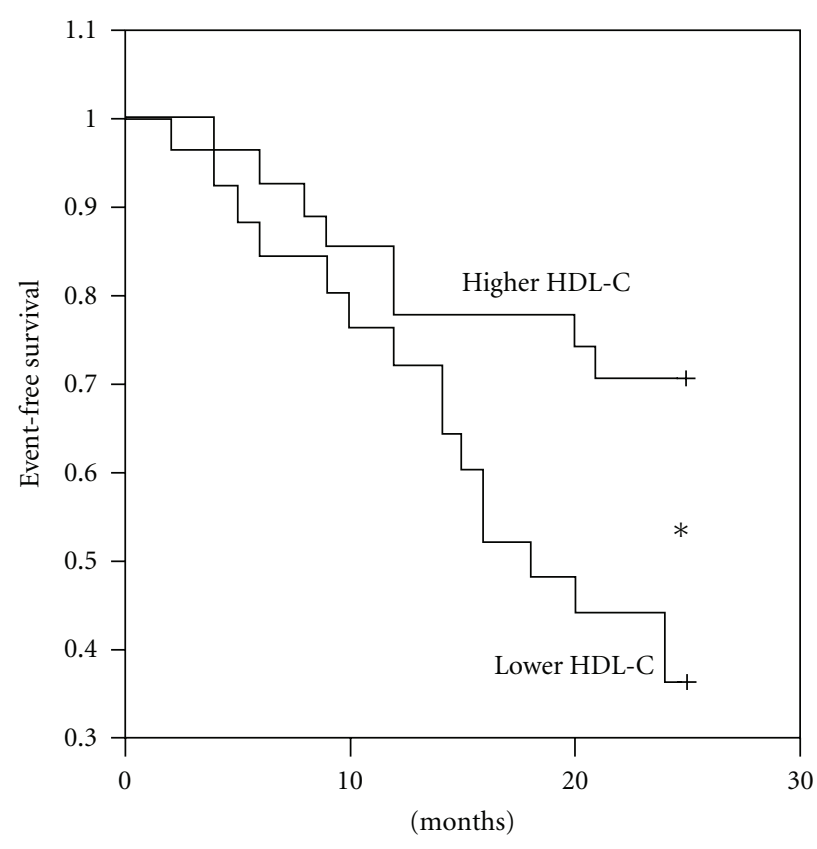

FIGURE 6: Kaplan-Meier analysis on diabetic hemodialysis patients showed that patients with higher HDL-C AI enjoy cardiovascular event-free lives longer than those with lower HDL-C. Log-rank test denoted that there was a significant difference in cardiovascular events between the two subgroups.

\section{Discussion}

Cardiovascular diseases are a major cause of death in hemodialysis patients [23]. Early detection of cardiovascular diseases and prevention of cardiovascular events appear important also in hemodialysis patients. We have previously reported that cardio-ankle vascular index, another index of arterial stiffness, is useful for this purpose, because it provides the cutoff value of 7.55 to predict the presence of cardiovascular diseases in hemodialysis patients [30]. Consistent with Covic et al. [33], our study suggests that the measurement of AI is also useful to screen the presence of cardiovascular diseases even if asymptomatic. ROC analysis showed that the cutoff value of 87 for AI predicted the occurrence of cardiovascular events in nondiabetic hemodialysis patients. Together, the present findings indicate that nondiabetic hemodialysis patients possessing AI over 87 should receive further medical examinations for the presence of cardiovascular diseases and implicate a critical role of central blood pressure in the development of cardiovascular events in hemodialysis patients.

Our results constitute a novel finding that the shortage in height contributed to total death in the present study. Shortness in height would reduce the time required for reflection wave to reach ascending aorta, thereby increasing AI [13]. Indeed, stepwise regression analyses demonstrated that the determinant of AI consisted of the height in both diabetic and nondiabetic groups. However, KaplanMeier analysis failed to attain statistical significance when a comparison was made to mortality between tall and short patients. Although precise reasons for the discrepancy are not 
readily apparent from the present study, several confounding factors could be involved. Smokers prevailed in the tall patients (16 smokers) more than short ones (7 smokers). ASCOT-CAFÉ study demonstrated the advantage of lower AI for better cardiovascular outcomes in hypertensive patients [9]. As discussed (bide infra), AI measured in the sitting position, however, may be inappropriately low in diabetic group possibly due to the presence of neuropathy [14]. Taken together, these observations could account for that the shortness of height, an important determinant of AI, but not AI itself, was picked up as a risk factor of mortality. It is tempting to speculate that patients with short height are exposed to high AI and high central blood pressure, even if brachial blood pressure is controlled similarly to tall patients. Indeed, Asians who suffer more cerebrovascular attacks than coronary heart disease are generally shorter than Caucasians [18]. The difference in body size as well as racial background may underlie high prevalence of stroke in Asia. Alternatively, the treatment goal of blood pressure for hypertensive patients should be individualized and adjusted by taking the influence of height on central blood pressure into consideration [34]. Taken together, these observations raise the possibility that blood pressure should be more carefully controlled for patients with short height such as women [35].

Several factors such as age, LVH, and arterial stiffness have been reported to predict cardiovascular prognosis.It seems controversial that AI constitutes a good predictor of cardiovascular complications in diabetes [10]. Indeed, AI was selected as an important contributor to cardiovascular events for nondiabetic but not diabetic patients in the present study. The present results support a recent investigation implicating that pulse pressure predicted cardiovascular survival in nondiabetic, but not diabetic hemodialysis patients [36]. In this study, however, LVH was selected as one of contributors to cardiovascular events in diabetes. Our previous data demonstrated that AI positively correlated to left ventricular mass Index in patients with nondiabetic CKD [27]. Our recent data demonstrated that patients with diabetic nephropathy manifested greater decreases in AI, brachial and central blood pressures when their postures alter from supine to sitting position than those with nondiabetic CKD [14]. The patient with diabetic nephropathy commonly suffers neuropathy as well [37]. Diabetic neuropathy could fade appropriate vasoconstriction following to postural changes, resulting in a large decrease in reflection wave. Alternatively, sleep may markedly increase central blood pressure in diabetes with neuropathy and facilitate the development of LVH. Collectively, blood pressure control at night in lying position would be important to prevent $\mathrm{LVH}$, cardiovascular events, and death, especially in diabetes.

Atherosclerotic cardiovascular diseases constitute a major cause of death in diabetic patients [38]. Frequently, type II diabetes has already manifested atherosclerotic vascular lesions at the time of diagnosis, and the presence of atherosclerosis makes their survival shorter and may increase total medical cost. Thus, the prevention and detection of atherosclerosis and cardiovascular complications in diabetes should be of clinical significance.
In addition to glycemic control, lipid-lowering drugs, and renin-angiotensin inhibitors appear as promising therapeutic tools to retard the development of atherosclerosis in diabetes $[39,40]$. In consistent, this study demonstrated that diabetes was a significant contributor to cardiovascular events. The usage of RAS inhibitors and statins was similar between diabetic and nondiabetic group, suggesting that the differences in medication between groups might have played a small role [39-41]. Our recent data indicated that oxidized LDL was higher in diabetic patients than those without diabetes [26]. Thus, the present results are compatible with recent investigations which revealed that oxidative stress underlies diabetic nephropathy [42], and suggest that oxidative stress is also involved in the pathogenesis of cardiovascular diseases in diabetes.

Chronic kidney disease (CKD) is characterized by oxidative stress $[43,44]$. In the case of hemodialysis, the contact of blood cells with dialyzer membrane may generate more oxidative stress to the patient. We have previously demonstrated that oxidized LDL was sky high in hemodialysis patients [45]. Our recent data suggest that oxidative stress induces insulin resistance, which is an important cardiovascular risk factor in hemodialysis patients [26]. It is easy to expect that the superimposition of exogenous oxidative stress on endogenous one would increase cardiovascular risk. Oxidative stress also causes the damage of DNA and histones, possibly underlying the pathogenesis of cancer [46]. Consequently, the present results that smoking strongly contributed to total and cardiovascular mortality are consistent with the above, and suggest that the prohibition of smoking is encouraged especially for CKD patients.

HDL-C plays a pivotal role in lipid metabolism by uptaking redundant cholesterol and carrying it back to liver for degradation. Recent investigations reveal that HDLC possesses strong antioxidant activity [47, 48]. In the present study, low HDL-C was selected as the contributor to cardiovascular events when diabetic as well as whole patients were examined. Our previous data indicated that higher oxidized LDL resulted in lower ankle-brachial pressure index in hemodialysis patients [45], and that hemodialysis patients with low ABI exhibit a strong tendency to develop cardiovascular events [26]. Our data seem compatible with those by Nishizawa et al. that non-HDL-C such as intermediate density lipoprotein cholesterol is highly atherogenic in hemodialysis patients [49] and suggest that in patients with CKD, the efforts to maintain HDL-C level such as aerobic exercise are more important for vascular health than those without renal dysfunction.

This study shares several limitations. Although further larger scale study should be required before drawing conclusion, the present data suggest that the values of AI in sitting position as a predictor of cardiovascular prognosis in hypertensive patients with diabetic nephropathy might be less than our expectation. Prospective studies that utilize AI measured in lying position should be required to examine $\mathrm{AI}$ as a predictor of cardiovascular prognosis in diabetes. Second, the usage of echocardiographic LVH as a risk factor might give differing results because electrocardiographic LVH does not always reflect echocardiographic LVH [29]. 
Thirdly, although TG was higher in diabetes, HDL-C was similar between the 2 groups in this study. However, TG did not contribute to either cardiovascular death or events. While the reasons for this discrepancy are not readily clear, we should note that TG levels were not so high in diabetes and that blood glucose was fairly controlled, presumably accounting for this. Fourthly, nonclassical risk factors such as albumin and anemia were not selected as a significant contributor to mortality or cardiovascular events. We should note that the patients enrolled in the present study were relatively young and shared well nutrition. Finally, oxidative stress and central hypertension may cause crosstalk to each other. Oxidative stress should increase central blood pressure by attenuating the actions of nitric oxide on artery, and high central blood pressure could induce oxidative stress de novo.

In conclusion, the present observations provide new evidence that shortness in height independently contributes to mortality in hemodialysis patients.

\section{Acknowledgments}

The authors thank Eriko Oshima, Sachiko Aida, Tadashi Uchino, Shingo Iwasaki, and Kenji Takahashi for their excellent technical assistance. They declare that the results presented in this paper have not been published previously in whole or part, except in abstract format. Parts of the data presented in this manuscript were presented in 51st annual meeting of Japanese Association of Dialysis Therapy.

\section{References}

[1] M. Yambe, H. Tomiyama, J. Yamada et al., "Arterial stiffness and progression to hypertension in Japanese male subjects with high normal blood pressure," Journal of Hypertension, vol. 25, no. 1, pp. 87-93, 2007.

[2] T. Mimura, T. Takenaka, Y. Kanno, K. Moriwaki, H. Okada, and H. Suzuki, "Vascular compliance is secured under angiotensin inhibition in non-diabetic chronic kidney diseases," Journal of Human Hypertension, vol. 22, no. 1, pp. 3847, 2008.

[3] T. Takenaka, K. Kobayashi, and H. Suzuki, "Pulse wave velocity as an indicator of arteriosclerosis in hemodialysis patients," Atherosclerosis, vol. 176, no. 2, pp. 405-409, 2004.

[4] J. Blacher, A. P. Guerin, B. Pannier, S. J. Marchais, M. E. Safar, and G. M. London, "Impact of aortic stiffness on survival in end-stage renal disease," Circulation, vol. 99, no. 18, pp. 24342439, 1999.

[5] K. Cruickshank, L. Riste, S. G. Anderson, J. S. Wright, G. Dunn, and R. G. Gosling, "Aortic pulse-wave velocity and its relationship to mortality in diabetes and glucose intolerance: an integrated index of vascular function?" Circulation, vol. 106, no. 16, pp. 2085-2090, 2002.

[6] G. M. London, J. Blacher, B. Pannier, A. P. Guérin, S. J. Marchais, and M. E. Safar, "Arterial wave reflections and survival in end-stage renal failure," Hypertension, vol. 38, no. 3, pp. 434-438, 2001.

[7] T. Takenaka, T. Mimura, Y. Kanno, Y. Ohno, and H. Suzuki, "Arterial wave reflection is elevated in evening hemodialysis patients," Clinical and Experimental Hypertension, vol. 30, no. 3-4, pp. 173-181, 2008.
[8] S. Laurent, P. Boutouyrie, R. Asmar et al., "Aortic stiffness is an independent predictor of all-cause and cardiovascular mortality in hypertensive patients," Hypertension, vol. 37, no. 5, pp. 1236-1241, 2001.

[9] B. Williams, P. S. Lacy, S. M. Thom et al., "Differential impact of blood pressure-lowering drugs on central aortic pressure and clinical outcomes: principal results of the Conduit Artery Function Evaluation (CAFE) study," Circulation, vol. 113, no. 9, pp. 1213-1225, 2006.

[10] P. S. Lacy, D. G. O’Brien, A. G. Stanley, M. M. Dewar, P. P. R. Swales, and B. Williams, "Increased pulse wave velocity is not associated with elevated augmentation index in patients with diabetes," Journal of Hypertension, vol. 22, no. 10, pp. 19371944, 2004.

[11] J. Westerbacka, E. Leinonen, J. T. Salonen et al., "Increased augmentation of central blood pressure is associated with increases in carotid intima-media thickness in type 2 diabetic patients," Diabetologia, vol. 48, no. 8, pp. 1654-1662, 2005.

[12] W. W. Nichols and B. M. Singh, "Augmentation index as a measure of peripheral vascular disease state," Current Opinion in Cardiology, vol. 17, no. 5, pp. 543-551, 2002.

[13] S. J. Zieman, V. Melenovsky, and D. A. Kass, "Mechanisms, pathophysiology, and therapy of arterial stiffness," Arteriosclerosis, Thrombosis, and Vascular Biology, vol. 25, no. 5, pp. 932943, 2005.

[14] T. Takenaka, T. Sato, H. Hoshi et al., "Zigzagged augmentation index in diabetes," Clinical and Experimental Hypertension, vol. 31, no. 8, pp. 657-668, 2009.

[15] T. A. Paajanen, N. K. J. Oksala, P. Kuukasjärvi, and P. J. Karhunen, "Short stature is associated with coronary heart disease," European Heart Journal, vol. 31, no. 14, pp. 1802$1809,2010$.

[16] Y.-M. Song and J. Sung, "Adult height and the risk of mortality in south Korean women," American Journal of Epidemiology, vol. 168, no. 5, pp. 497-505, 2008.

[17] Y.-M. Song, G. D. Smith, and J. Sung, "Adult height and causespecific mortality: a large prospective study of South Korean men," American Journal of Epidemiology, vol. 158, no. 5, pp. 479-485, 2003.

[18] C. M. Y. Lee, F. Barzi, M. Woodward et al., "Adult height and the risks of cardiovascular disease and major causes of death in the Asia-Pacific region," International Journal of Epidemiology, vol. 38, no. 4, pp. 1060-1071, 2009.

[19] K. Silventoinen, J. Kaprio, M. Koskenvuo, and E. Lahelma, "The association between body height and coronary heart disease among Finnish twins and singletons," International Journal of Epidemiology, vol. 32, no. 1, pp. 78-82, 2003.

[20] A. L. Batide-Alanore, D.-A. Trégouët, C. Sass, G. Siest, S. Visvikis, and L. Tiret, "Family study of the relationship between height and cardiovascular risk factors in the STANISLAS cohort," International Journal of Epidemiology, vol. 32, no. 4, pp. 607-614, 2003.

[21] C. Langenberg, R. Hardy, D. Kuh, and M. E. J. Wadsworth, "Influence of height, leg and trunk length on pulse pressure, systolic and diastolic blood pressure," Journal of Hypertension, vol. 21, no. 3, pp. 537-543, 2003.

[22] K. Miura, H. Nakagawa, Y. Ohashi et al., "Japan Arteriosclerosis Longitudinal Study Group. Four blood pressure indexes and the risk of stroke and myocardial infarction in Japanese men and women," Circulation, vol. 119, no. 14, pp. 1892-1898, 2009.

[23] M. J. Sarnak, "Cardiovascular complications in chronic kidney disease," American Journal of Kidney Diseases, vol. 41, no. 5, supplement, pp. 11-17, 2003. 
[24] T. Nakamura, Y. Kanno, T. Takenaka et al., "An angiotensin receptor blocker reduces the risk of congestive heart failure in elderly hypertensive patients with renal insufficiency," Hypertension Research, vol. 28, no. 5, pp. 415-423, 2005.

[25] Patient Registration Committee, "Japanese Society for Dialysis Therapy. An overview of regular dialysis treatment in Japan (as of 31 December 2002)," Therapeutic Apheresis and Dialysis, vol. 8, no. 5, pp. 358-382, 2004.

[26] T. Takenaka, Y. Kanno, Y. Ohno, and H. Suzuki, "Key role of insulin resistance in vascular injury among hemodialysis patients," Metabolism: Clinical and Experimental, vol. 56, no. 2, pp. 153-159, 2007.

[27] T. Takenaka, T. Mimura, Y. Kanno, and H. Suzuki, "Qualification of arterial stiffness as a risk factor to the progression of chronic kidney diseases," American Journal of Nephrology, vol. 25, no. 5, pp. 417-424, 2005.

[28] K. Takazawa, H. Kobayashi, N. Shindo, N. Tanaka, and A. Yamashina, "Relationship between radial and central aeterial pulse wave and evaluation of central aortic pressure using the radial arterial pulse wave," Hypertension Research, vol. 30, no. 3, pp. 219-228, 2007.

[29] J. Sundström, L. Lind, J. Ärnlöv, B. Zethelius, B. Andrén, and H. O. Lithell, "Echocardiographic and electrocardiographic diagnoses of left ventricular hypertrophy predict mortality independently of each other in a population of elderly men," Circulation, vol. 103, no. 19, pp. 2346-2351, 2001.

[30] T. Takenaka, H. Hoshi, N. Kato et al., "Cardio-ankle vascular index to screen cardiovascular diseases in patients with endstage renal diseases," Journal of Atherosclerosis and Thrombosis, vol. 15, no. 6, pp. 339-344, 2008.

[31] T.-M. Lyyra, E. Leskinen, and E. Heikkinen, "A cohort study found good respiratory, sensory and motor functions decreased mortality risk in older people," Journal of Clinical Epidemiology, vol. 58, no. 5, pp. 509-516, 2005.

[32] D. A. Goodkin, J. L. Bragg-Gresham, K. G. Koenig et al., "Association of comorbid conditions and mortality in hemodialysis patients in Europe, Japan, and the United States: the Dialysis Outcomes and Practice Patterns Study," Journal of the American Society of Nephrology, vol. 14, no. 12, pp. 32703277, 2003.

[33] A. Covic, A. A. Haydar, P. Bhamra-Ariza, P. Gusbeth-Tatomir, and D. J. Goldsmith, "Aortic pulse wave velocity and arterial wave reflections predict the extent and severity of coronary artery disease in chronic kidney disease patients," Journal of Nephrology, vol. 18, no. 4, pp. 388-396, 2005.

[34] S. J. Marchais, A. P. Guerin, B. M. Pannier, B. I. Levy, M. E. Safar, and G. M. London, "Wave reflections and cardiac hypertrophy in chronic uremia: influence of body size," Hypertension, vol. 22, no. 6, pp. 876-883, 1993.

[35] C. D. Gatzka, B. A. Kingwell, J. D. Cameron et al., "Gender differences in the timing of arterial wave reflection beyond differences in body height," Journal of Hypertension, vol. 19, no. 12, pp. 2197-2203, 2001.

[36] M. Tozawa, K. Iseki, C. Iseki, and S. Takishita, "Pulse pressure and risk of total mortality and cardiovascular events in patients on chronic hemodialysis," Kidney International, vol. 61, no. 2, pp. 717-726, 2002.

[37] J. A. Meinhold, E. Maslowska-Wessel, R. Bender, and P. T. Sawicki, "Low prevalence of cardiac autonomic neuropathy in Type 1 diabetic patients without nephropathy," Diabetic Medicine, vol. 18, no. 8, pp. 607-613, 2001.

[38] G. L. Bakris, M. Williams, L. Dworkin et al., "Preserving renal function in adults with hypertension and diabetes: a consensus approach," American Journal of Kidney Diseases, vol. 36, no. 3, pp. 646-661, 2000.

[39] A. Keech, D. Colquhoun, J. Best et al., "LIPID Study Group. Secondary prevention of cardiovascular events with longterm pravastatin in patients with diabetes of impaired fasting glucose: results from the LIPID trial," Diabetes Care, vol. 26, no. 10, pp. 2713-2721, 2003.

[40] H. Suzuki, Y. Kanno, S. Sugahara et al., "Effect of angiotensin receptor blockers on cardiovascular events in patients undergoing hemodialysis: an open-label randomized controlled trial," American Journal of Kidney Diseases, vol. 52, no. 3, pp. 501-506, 2008.

[41] H. Miyashita, A. Aizawa, J. Hashimoto et al., "Cross-sectional characterization of all classes of antihypertensives in terms of central blood pressure in Japanese hypertensive patients," American Journal of Hypertension, vol. 23, no. 3, pp. 260-268, 2010.

[42] J. M. Forbes, M. T. Coughlan, and M. E. Cooper, "Oxidative stress as a major culprit in kidney disease in diabetes," Diabetes, vol. 57, no. 6, pp. 1446-1454, 2008.

[43] T. Takenaka, H. Takane, H. Okada, Y. Ohno, and H. Suzuki, "Long-term effects of calcium antagonists on augmentation index in hypertensive patients with chronic kidney diseases," NDT Plus, vol. 2, no. 2, pp. 192-193, 2009.

[44] N. J. Brunskill, "Albumin handling by proximal tubular cells: mechanisms and mediators," Nephrology Dialysis Transplantation, vol. 15, supplement, pp. 39-40, 2000.

[45] T. Takenaka, K. Takahashi, T. Kobayashi, E. Oshima, S. Iwasaki, and H. Suzuki, "Oxidized low density lipoprotein (Ox-LDL) as a marker of atherosclerosis in hemodialysis (HD) patients," Clinical Nephrology, vol. 58, no. 1, pp. 33-37, 2002.

[46] R. B. Parmigiani, W. S. Xu, G. Venta-Perez et al., "HDAC6 is a specific deacetylase of peroxiredoxins and is involved in redox regulation," Proceedings of the National Academy of Sciences of the United States of America, vol. 105, no. 28, pp. 9633-9638, 2008.

[47] R. Ross and B. Dodet, "Atherosclerosis is an inflammatory disease," The New England Journal of Medicine, vol. 340, no. 2, pp. 115-126, 1999.

[48] N. D. Vaziri, M. Navab, and A. M. Fogelman, "HDL metabolism and activity in chronic kidney disease," Nature Reviews Nephrology, vol. 6, no. 5, pp. 287-296, 2010.

[49] Y. Nishizawa, T. Shoji, R. Kakiya et al., "Non-high-density lipoprotein cholesterol (non-HDL-C) as a predictor of cardiovascular mortality in patients with end-stage renal disease," Kidney International, Supplement, vol. 63, no. 84, pp. S117S120, 2003. 


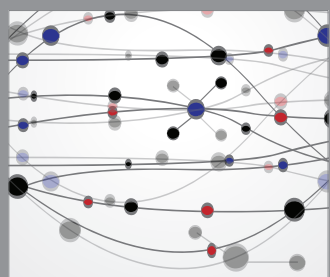

The Scientific World Journal
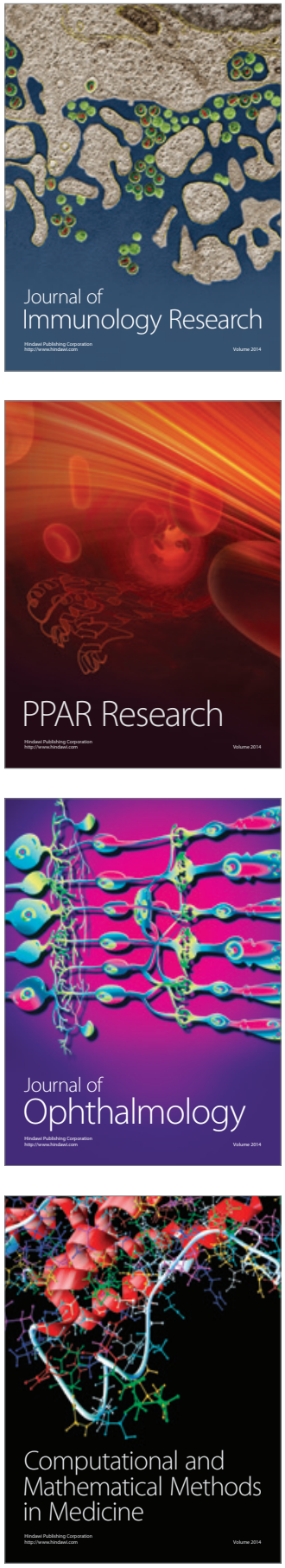

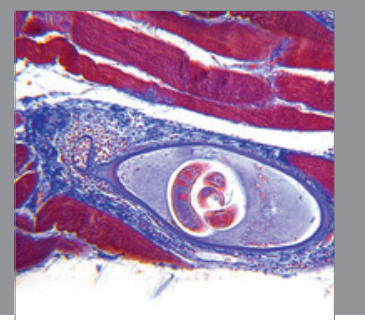

Gastroenterology

Research and Practice
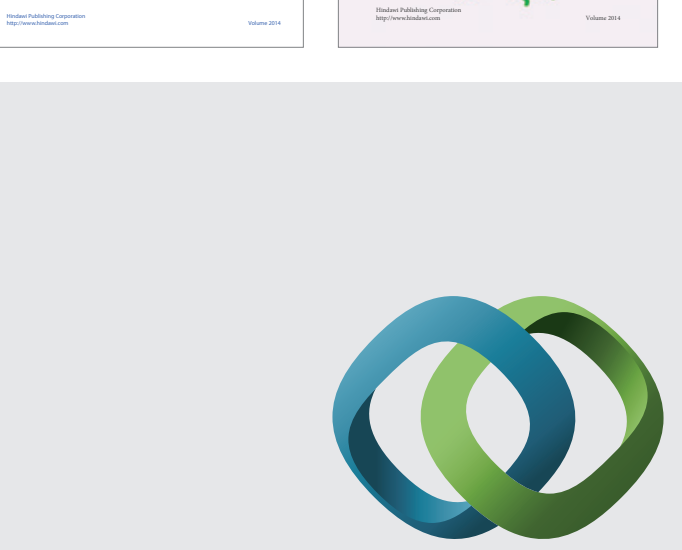

\section{Hindawi}

Submit your manuscripts at

http://www.hindawi.com
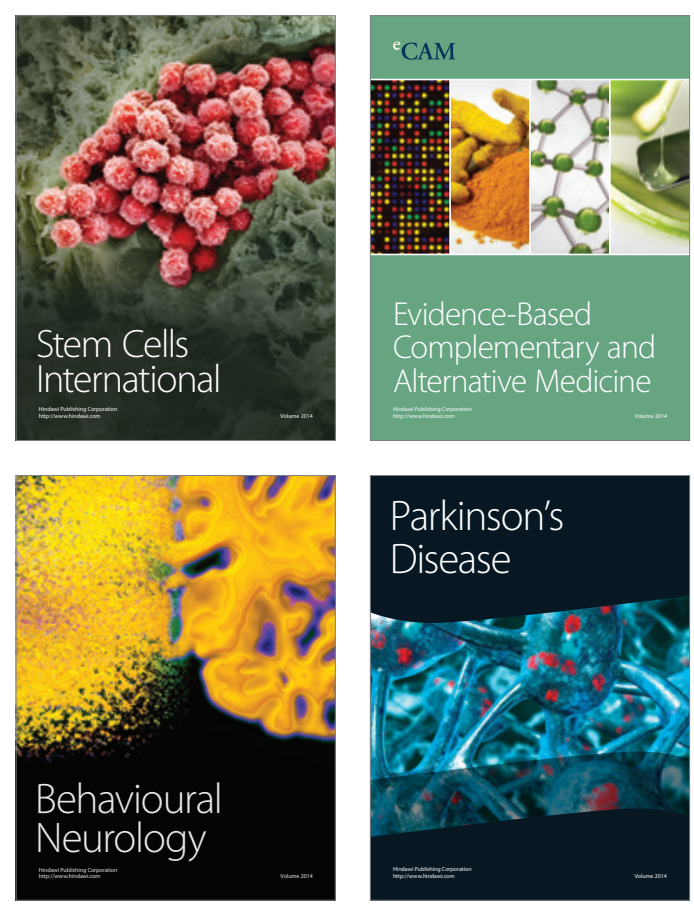

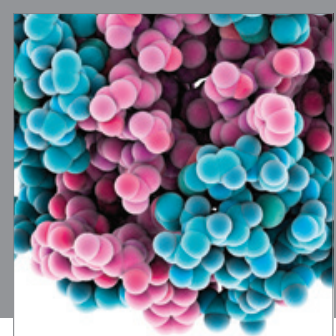

Journal of
Diabetes Research

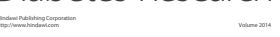

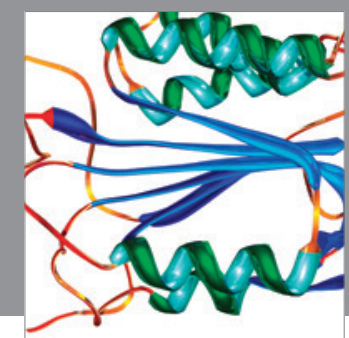

Disease Markers
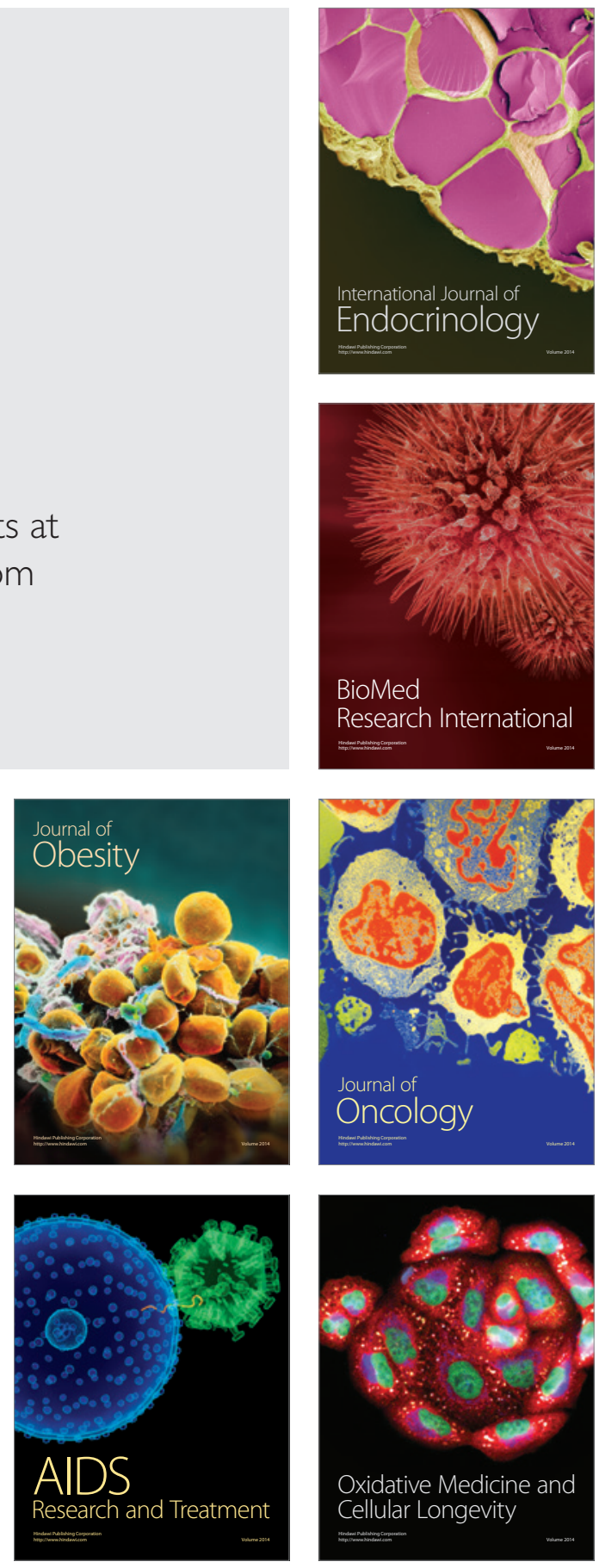